\title{
Data mining tools for Salmonella characterization: application to gel-based fingerprinting analysis
}

\author{
Wen Zou ${ }^{1 *}$, Hailin Tang', Weizhong Zhao ${ }^{1}$, Joe Meehan', Steven L Foley², Wei-Jiun Lin ${ }^{3}$, Hung-Chia Chen ${ }^{1,4}$, \\ Hong Fang ${ }^{5}$, Rajesh Nayak², James J Chen ${ }^{1}$ \\ From Tenth Annual MCBIOS Conference. Discovery in a sea of data \\ Columbia, MO, USA. 5-6 April 2013
}

\begin{abstract}
Background: Pulsed field gel electrophoresis (PFGE) is currently the most widely and routinely used method by the Centers for Disease Control and Prevention (CDC) and state health labs in the United States for Salmonella surveillance and outbreak tracking. Major drawbacks of commercially available PFGE analysis programs have been their difficulty in dealing with large datasets and the limited availability of analysis tools. There exists a need to develop new analytical tools for PFGE data mining in order to make full use of valuable data in large surveillance databases.

Results: In this study, a software package was developed consisting of five types of bioinformatics approaches exploring and implementing for the analysis and visualization of PFGE fingerprinting. The approaches include PFGE band standardization, Salmonella serotype prediction, hierarchical cluster analysis, distance matrix analysis and two-way hierarchical cluster analysis. PFGE band standardization makes it possible for cross-group large dataset analysis. The Salmonella serotype prediction approach allows users to predict serotypes of Salmonella isolates based on their PFGE patterns. The hierarchical cluster analysis approach could be used to clarify subtypes and phylogenetic relationships among groups of PFGE patterns. The distance matrix and two-way hierarchical cluster analysis tools allow users to directly visualize the similarities/dissimilarities of any two individual patterns and the inter- and intra-serotype relationships of two or more serotypes, and provide a summary of the overall relationships between user-selected serotypes as well as the distinguishable band markers of these serotypes. The functionalities of these tools were illustrated on PFGE fingerprinting data from PulseNet of CDC.

Conclusions: The bioinformatics approaches included in the software package developed in this study were integrated with the PFGE database to enhance the data mining of PFGE fingerprints. Fast and accurate prediction makes it possible to elucidate Salmonella serotype information before conventional serological methods are pursued. The development of bioinformatics tools to distinguish the PFGE markers and serotype specific patterns will enhance PFGE data retrieval, interpretation and serotype identification and will likely accelerate source tracking to identify the Salmonella isolates implicated in foodborne diseases.
\end{abstract}

\section{Background}

Food safety remains an important concern due in part to the globalization of food supply and foodborne illnesses create an important public health burden in the United States. CDC data indicates that nearly 48 million

\footnotetext{
* Correspondence: wen.zou@fda.hhs.gov

'Division of Bioinformatics and Biostatistics, U.S. Food and Drug

Administration, Jefferson, Arkansas, USA

Full list of author information is available at the end of the article
}

people become ill, 128,000 are hospitalized, and 3,000 die due to foodborne illnesses each year, and nontyphoidal Salmonella enterica is one of the leading causes of illnesses among the top 31 known foodborne pathogens [1]. The characteristics of Salmonella infections has changed over time, including changes in the frequency of antimicrobial-resistant Salmonella subtypes implicated and the frequency of different serotypes among isolates associated with human infections [2]. 
Multiple phenotypic and genotypic Salmonella subtyping methods have been developed to efficiently detect the cases of human salmonellosis [3]. These methods include traditional phenotype-based approaches such as serotyping [4]; genotype-based methods such as Pulsed Field Gel Electrophoresis (PFGE) [3,5]; DNA sequencebased methods including DNA microarray analysis, multi-locus sequence typing (MLST) [6,7], multi-locus variable-number tandem repeat analysis (MLVA) $[8,9]$ and next-generation sequencing (NGS) [10-14]. Each of the subtyping approaches has been applied in Salmonella outbreak strain identification and source tracking; however they each have their own strengths and weaknesses in terms of sensitivity, cost, speed, and robustness.

Large amounts of molecular subtyping data have been generated by academia, private companies and government agencies. Along with the development of new technologies, it is anticipated that new analytical methods will be applied more often in combination with the conventional assays to characterize and subtype foodborne isolates, therefore, enhancing the current food safety and regulatory science paradigm [15]. Facing the large amount of emerging data and technologies, one of the major challenges is the data management, storage, analysis and retrieval, and how to build up the connections and communication for data developed by various subtyping methods. Data mining seeks to find new interesting patterns and relationships in huge amounts of data. Data mining involves the bioinformatics approaches that combine biological data using computational tools and statistical methods to analyze, summarize and transform data into useful information to improve food safety. Such a systematic approach facilitates the extraction and correlation of patterns of knowledge that is implicit in the stored databases.

PFGE is currently the most widely and routinely used molecular subtyping method by CDC and state health labs in the US for Salmonella surveillance and outbreak investigation [16]. Although PFGE provides lessdetailed genetic information than NGS and other DNA sequence-based methods, it has been successfully used for over twenty years to type Salmonella from human patients, foods, and food animal sources because of its discriminatory power, low cost and high reproducibility $[3,5]$. PulseNet (http://www.cdc.gov/pulsenet), the CDC's molecular surveillance network used for foodborne infections, has the largest and most rich Salmonella subtyping database in the world, storing more than 350,000 PFGE patterns of more than 500 serotypes since 1996 [17]. Data mining of this valuable database will provide resources to study the ecology, epidemiology, transmission, and evolution of the emerging Salmonella serotypes.
Several commercial software applications have been used to analyze PFGE data, such as BioNumerics (Applied Maths, Inc., Austin, TX), GelCompar II (Applied Maths, Kortrijk, Belgium) and Fingerprinting II version 3 (Bio-Rad, Hercules, USA). BioNumerics is the default software in PulseNet standard protocol $[18,19]$ and has been widely used in PulseNet participating laboratories and other public health laboratories that perform PFGE subtyping for bacterial foodborne pathogens for surveillance and outbreak investigations. These softwares are currently used to analyze PFGE gel images to generate dendrograms for clustering PFGE patterns from different strains of foodborne pathogens. No other methodologies or commercial tools are applicable on PFGE data except for the cluster analysis, which limits the usage of this subtyping technology in understanding the genetic diversities of foodborne bacteria. In addition, BioNumerics and other software have limitations on dealing with large number of samples (less than 20,000 patterns for Bionumerics), which is an obstacle for meta-analysis of the PFGE data and data mining.

In this study, in order to systematically investigate and characterize PFGE patterns of Salmonella isolates, BACPAK knowledgebase was created and systematic approaches assembled to build up a functional software package for PFGE data mining. The approaches include PFGE band standardization, Salmonella serotype prediction, hierarchical cluster analysis, distance matrix analysis and two-way hierarchical cluster analysis. The development of this software package and the application of its approaches provide a better understanding of Salmonella genetic diversity and epidemiology, and contribute to PFGE-based characterization and surveillance of Salmonella isolates in outbreak investigations.

\section{Implementation}

Bacterial pathogen knowledgebase (BACPAK) construction and PFGE database

An integrated genomic bacterial pathogen knowledgebase (BACPAK) is being constructed and housed at the NCTR. As an information system which aims to support the research on foodborne bacterial pathogen detection, characterization and outbreak investigation, BACPAK integrates investigational data from NCTR and other government agencies as well as expert-curated published data on foodborne bacterial pathogens (Table 1). As part of the BACPAK knowledgebase data composition, a total of 45,923 XbaI-PFGE patterns of Salmonella enterica isolates were collected in the PFGE database established in our previous work [20] (Table 1). These patterns were randomly selected within each of the 32 most frequent serotypes from all the submissions from human sources 
Table 1 The data composition in BACPAK and Salmonella PFGE fingerprints database.

\begin{tabular}{|c|c|}
\hline \multicolumn{2}{|c|}{ BACPAK } \\
\hline Antimicribial susceptibility test & 767 \\
\hline Antimicribial resistant gene PCR & 462 \\
\hline Plasmid sequence information & 34 \\
\hline PFGE & 45,923 \\
\hline \multicolumn{2}{|c|}{ PFGE Database } \\
\hline Serotypes & Number of patterns \\
\hline Agona & 1954 \\
\hline Braenderup & 2008 \\
\hline Enteritidis & 2338 \\
\hline Hadar & 1981 \\
\hline Heidelberg & 2114 \\
\hline 1 4, [5],12:i:- & 2281 \\
\hline Infantis & 2078 \\
\hline Javiana & 2102 \\
\hline Mississippi & 1999 \\
\hline Montevideo & 2041 \\
\hline Muenchen & 1970 \\
\hline Newport & 2005 \\
\hline Oranienburg & 1951 \\
\hline Paratyphi $B$ var. $L(+)$ tartrate+ & 2011 \\
\hline Poona & 1956 \\
\hline Saintpaul & 2252 \\
\hline Thompson & 2045 \\
\hline Typhi & 1941 \\
\hline Typhimurium & 2064 \\
\hline Typhimurium var. 5- & 2146 \\
\hline Anatum & 478 \\
\hline Bareilly & 426 \\
\hline Berta & 502 \\
\hline Derby & 393 \\
\hline Hartford & 531 \\
\hline Litchfield & 401 \\
\hline Mbandaka & 432 \\
\hline Panama & 516 \\
\hline Paratyphi A & 135 \\
\hline Schwarzengrund & 225 \\
\hline Senftenberg & 189 \\
\hline Stanley & 460 \\
\hline
\end{tabular}

to PulseNet from 2005 to 2010. The imported gel images were processed and analyzed by BioNumerics software (Applied Maths, Inc., Austin, TX, Version 6.0) according to the PulseNet protocol [19]. The band matching was performed at a trace-to-trace optimization value of $1.56 \%$ and a band position tolerance set at $1 \%$.

\section{PFGE band standardization}

Before analysis with the developed tools, the bands of all the PFGE patterns should be normalized. For example, when using Salmonella serotype prediction tool, the bands of tested Salmonella isolates should be normalized to band classes stored within the database, which are used in the development of training sets for the prediction tools. To accomplish this band normalization, the NCTR fixed band method [20] was implemented to standardize the band classes for cross-group analysis. In this method, the means of the band sizes of two adjacent bands of the training data was used as the standard to normalize the corresponding bands of each new sample. As an example, assume that the training data have a set of descending bands sized as s1, s2, s3, s4..., and the test sample consists of descending bands of $t 1, t 2, t 3$, $\mathrm{t} 4 \ldots$ if $\mathrm{t} 1 \leq(\mathrm{s} 1+\mathrm{s} 2) / 2, \mathrm{t} 1$ is normalized to $\mathrm{s} 2$, and if $\mathrm{t} 1>$ $(\mathrm{s} 1+\mathrm{s} 2) / 2$, it is adjusted to $\mathrm{s} 1$ [21]. A total of 39,830 PFGE patterns were band standardized and stored in the database [21].

\section{Salmonella serotype prediction from PFGE fingerprints}

Previous studies have reported two classification algorithms, Random Forest (RF) [22] and Support Vector Machine (SVM) [23], to predict Salmonella serotypes based on PFGE fingerprints [21,24]. The scripts of the algorithms were based on the packages "RandomForest" and "e1071" in R (version 2.12.1), respectively. Based on the prediction accuracies, the SVM algorithm was chosen to computerize the scripts as a practical tool for Salmonella prediction using PFGE fingerprints. The normalized database consisting of 39,830 patterns from 32 serotypes was used as the default standard and training set [21].

\section{Hierarchical cluster analysis}

The distances of any two of the standardized PFGE patterns were measured and hierarchical cluster analysis was pursued by the complete linkage method using "hcluster package" in R [25]. The scripts were converted to a computational tool provided in BACPAK.

\section{Distance matrix development and two-way hierarchical cluster analysis}

In the approach of distance matrix analysis, scripts were written in $\mathrm{R}$ to calculate the Jaccard Distance [26] of PFGE patterns for measuring the dissimilarity of PFGE inter- or intra-serotypes patterns. The color from blue to red indicted the values of the Jaccard Distance ranged from 0 to 1 . The scripts were computerized as a tool to identify the differences and relationships among the various Salmonella patterns within specific serotypes and among the targeted serotypes. 
In the two-way hierarchical cluster analysis, scripts were coded using $\mathrm{R}$ to calculate the average proportions of the bands present at every designated band location with values ranging from 0 to 1 to build the characteristic parameters of each target serotype. The hierarchical cluster analysis using the complete linkage was applied based on the dissimilarity measures of any two serotypes calculated by the Euclidean distance [27] of the characteristic parameters. The scripts were implemented to pursue a two-way clustering analysis of the PFGE patterns, in which both serotypes and band locations were clustered according to dissimilarity measures to simultaneously identify the associations between serotypes and band locations.

\section{Results and discussions}

To begin to address the need to develop improved analytical tools for PFGE analysis, a software package consisting of the integrated data mining techniques and the PFGE database was established and stored within NCTR's BACPAK. BACPAK is capturing and storing data including antimicrobial susceptibility data, plasmid sequence data, PCR data on antimicrobial resistance genes and PFGE data (Table 1). The PFGE database consisted of 45,923 semi-randomly selected PFGE patterns submitted to PulseNet from 2005 to 2010 (Table 1) [20]. Based on the statistics of the Salmonella Annual Report 2006 [2] and Salmonella Annual Summary Tables 2009 from CDC [28], isolates from the 32 serotypes represented in the BACPAK database comprised more than $80 \%$ of all Salmonella reported over the past 14 years in the US [21].

\section{The approach for PFGE band standardization}

Band normalization is the key point to allow the comparison from different dataset. Since BioNumerics was unstable to handle more than 20,000 PFGE patterns, the implemented NCTR fixed band method was especially useful for large dataset analysis. It showed higher accuracies when used to normalize PFGE bands for Salmonella serotype prediction in comparison to the conventional BioNumerics fixed band method [21], and made the meta-analysis available to clarify the inter- and intra-serotypes relationships in a large dataset [20]. In addition, NCTR fixed band method transferred the gel-imaged band class into certain digital parameters in the model, and normalized the bands of future candidates with no necessity to upload and save standard band class in BioNumerics [21].

\section{The prediction approach for Salmonella serotype prediction based on PFGE patterns}

The prediction algorithm was developed as described previously to identify Salmonella serotypes based on their PFGE patterns $[21,24]$. In these studies, the NCTR fixed band method coupled to the SVM classification produced the highest average predictive accuracies for serotype determination (96.1\%) [21]. Therefore, the SVM algorithm was coupled with NCTR standardization method and turned the $\mathrm{R}$ scripts which were implemented as a computational prediction tools installed in BACPAK.

Figure 1 shows the work chart of the prediction tool, which includes data normalization, followed by serotype prediction. A total of 39,830 PFGE patterns from the PFGE database were applied as the training dataset bound to the prediction tool [21]. The tool allows individual users to either test the tool with the data in BACPAK or upload their data in a proper format. The output result is presented in an Excel file showing the predicted serotypes.

As a test case, five Salmonella isolates (Table 2) were randomly selected from PulseNet but excluded from the training set of 39,830 PFGE patterns. The gel images were processed by BioNumerics software according to the PulseNet protocol [29]. The resultant file containing band presence/absence data was uploaded into the prediction tool in BACPAK. The output result is shown in Table 2. The five tested isolates had their serotypes correctly predicted.

The original prediction tools were developed by a supervised classification approach $[21,24]$. This approach focused on studying the association between PFGE

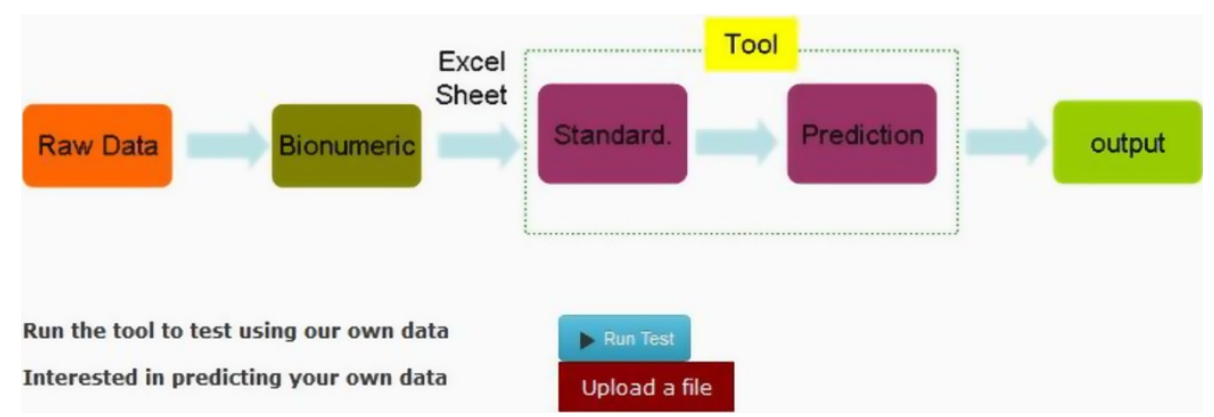

Figure 1 The flow chart of the tool for Salmonella serotype prediction. (The image was taken from BACPAK.) 
Table 2 Five selected test Salmonella isolates, the prediction results and the distinguished band markers by the twoway hierarchical cluster analysis tool for five serotype identification ("X" stands for band presence).

\begin{tabular}{|c|c|c|c|c|c|c|c|c|c|c|c|c|}
\hline Test Salmonella isolates & Predicted serotypes & Real serotypes & & & Dist & inguish & d band & marker & $5(\mathrm{~Kb})$ & & & \\
\hline & & & 32.8 & 84.9 & 127.2 & 160.1 & 168.3 & 223.2 & 237.5 & 373.9 & 411.8 & 459.9 \\
\hline T1: AK_0823200134 & I 4, [5],12:i:- & | 4, [5],12:i:- & & $x$ & & & & $x$ & & $x$ & & \\
\hline T2: AL_AL_8002189-06 & Thompson & Thompson & & & & & $x$ & & & & & $\mathrm{x}$ \\
\hline T3: CT_02024279 & Hadar & Hadar & & $x$ & & & & & $x$ & & & \\
\hline T4: MD_MD0622721 & Typhi & Typhi & $x$ & & & $x$ & & & & & & \\
\hline T5: TX_TXAML0902385 & Oranienburg & Oranienburg & & & $\mathrm{x}$ & & & & & & $x$ & \\
\hline
\end{tabular}

patterns and serotypes determined using traditional serological methods, and applying the information learned from the training set as the rules for prediction in the test set. The prediction accuracy was measured by applying the prediction model based on the training set to emulate the population of the future profiles to be analyzed. If the samples in the training set do not adequately represent the likely samples to be encountered in use, then bias may occur. The training set used in these studies represents greater than $80 \%$ of all the isolates reported to $\mathrm{CDC}$, therefore, the prediction tool should be able to predict most Salmonella serotypes. As such, this tool should be especially useful to predict the serotype of outbreak isolates before the conventional methods were carried out in a laboratory. The refinement of the predictive tool is an ongoing effort as additional PFGE data becomes available and is incorporated into the training dataset to improve the prediction accuracies.

\section{Hierarchical cluster analysis}

Unlike the supervised classification algorithm, hierarchical cluster analysis is unsupervised, where the samples are grouped into subsets based only on the pairwise similarities among their PFGE profiles without using serotype information [24]. As a case study, five test Salmonella isolates were added to the dataset of the 10,193 PFGE patterns of the five predicted serotypes (Table 2) which were retrieved from the PFGE database in BACPAK. The hierarchical analysis tool was applied on this dataset (10,198 PFGE patterns) and the dendrogram is shown in Figure 2. At the cutting threshold of 0.98 , all 10,198 patterns were grouped into 9 clusters (C1 to $\mathrm{C} 9$ ), and four

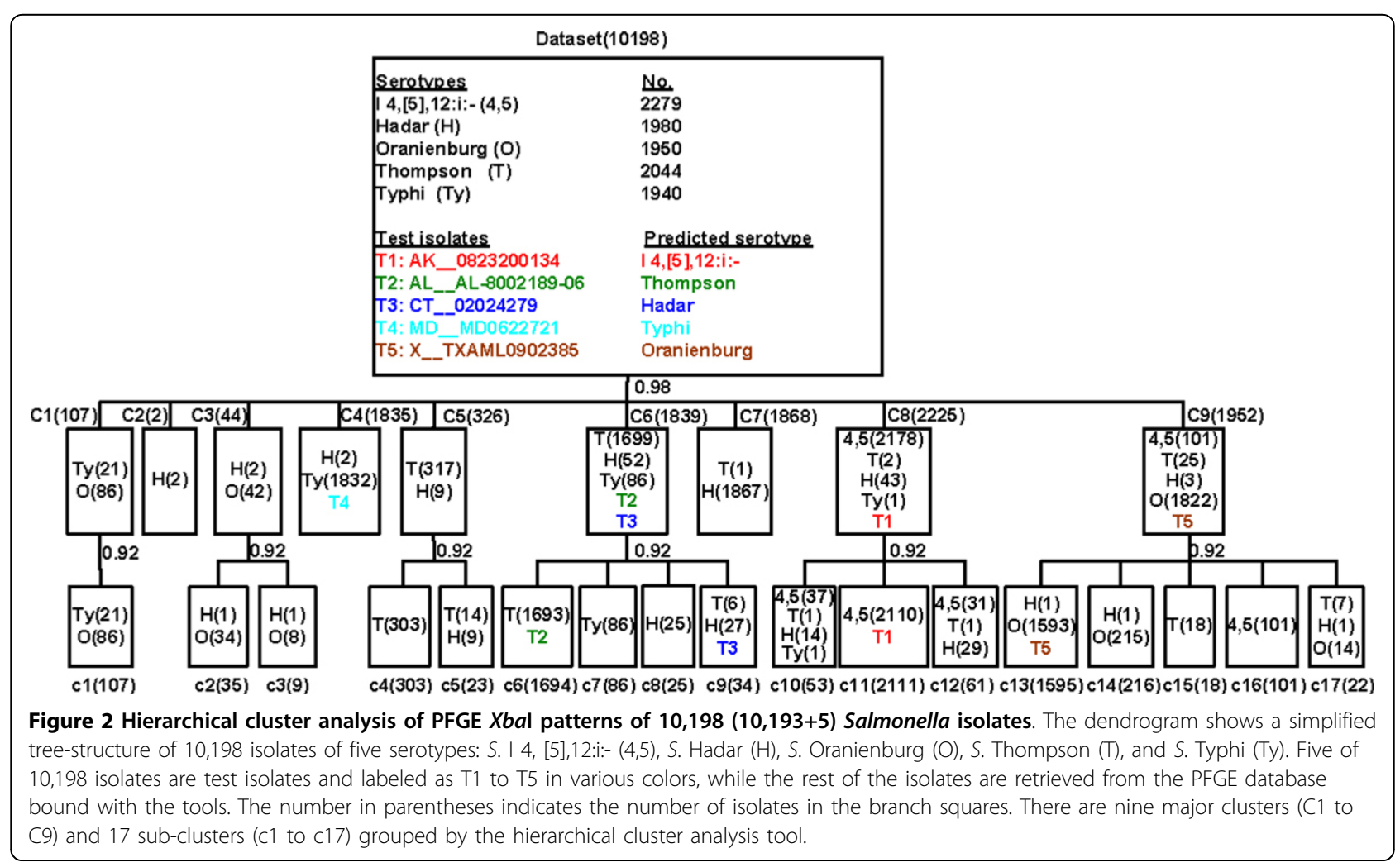


of five test samples were separated into four individual groups where the predicted serotypes of the four test isolates matched those of the majority samples in the same cluster (Figure 2). The exception was the isolate CT_02024279 whose serotype was correctly predicted as Hadar by the prediction tool (Table 2). Some subtypes were distinguished at this cutting threshold. For example, the predominant cluster of serotype Oranienburg was in C9 which harbored 1822 patterns of Oranienburg, while two subtypes were located in C1 (86 patterns of Oranienburg) and C3 (42 patterns of Oranienburg) (Figure 2). Serotype Thompson was found to have 1 predominant type (C6) and two subtypes (C5 and C9). When the cutting threshold decreased to 0.92 , more subtypes were distinguished (c1 to c17). C5 at threshold of 0.98 was further classified into two groups of $\mathrm{c} 4$ and $\mathrm{c} 5$ at threshold of 0.92 , and c4 had a pure composition of 303 Thompson strains. Test Salmonella isolates T2 and T3 in C6 was clarified into different groups of c6 (T2) and c9 (T3). At this cutting threshold, the predominant pure group of each of the five serotypes was identified (C4 for Typhi, c6 for Thompson, C7 for Hadar, c11 for I 4, [5],12:i:- and c13 for Oranienburg), and four of five test
Salmonella isolates (T1, T2, T4 and T5) were clustered together with the predominant groups of their corresponding serotypes, respectively. T3 was mis-classified with 27 isolates of same serotype Hadar in Thompson predominant group C6, indicating that this subgroup of Hadar isolates had close relationship with serotype Thompson. The tool of hierarchical cluster analysis allows users to distinguish the underlying phylogenetic structures or to discover new subtypes between various PFGE patterns. With the emergence of NGS and other sequencing technologies, the subtypes distinguished by hierarchical cluster analysis tool will be clarified in detail.

\section{Distinguishing serotype relationships: distance matrix and two-way hierarchical cluster analysis}

Distance matrix and two-way hierarchical cluster analysis provide users the appropriate methods to further distinguish the relationships among Salmonella serotypes. Five serotypes were selected to demonstrate the functionality of these tools. A total of 10,193 PFGE patterns belonging to the five serotypes (Table 2) were retrieved from the database and uploaded to the two tools. Figure 3 exhibited the heatmap of the distance matrix of 10,193 PFGE

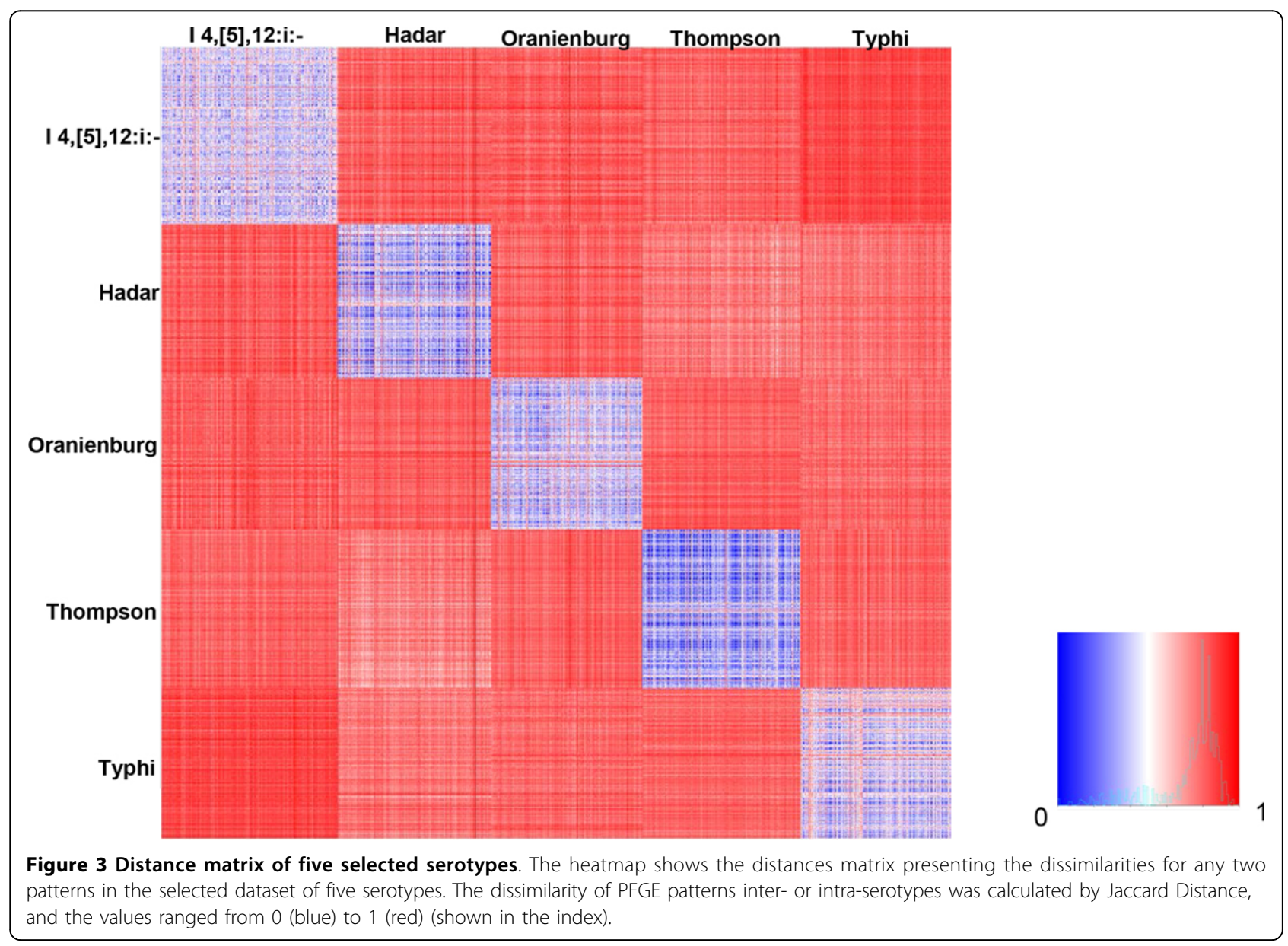




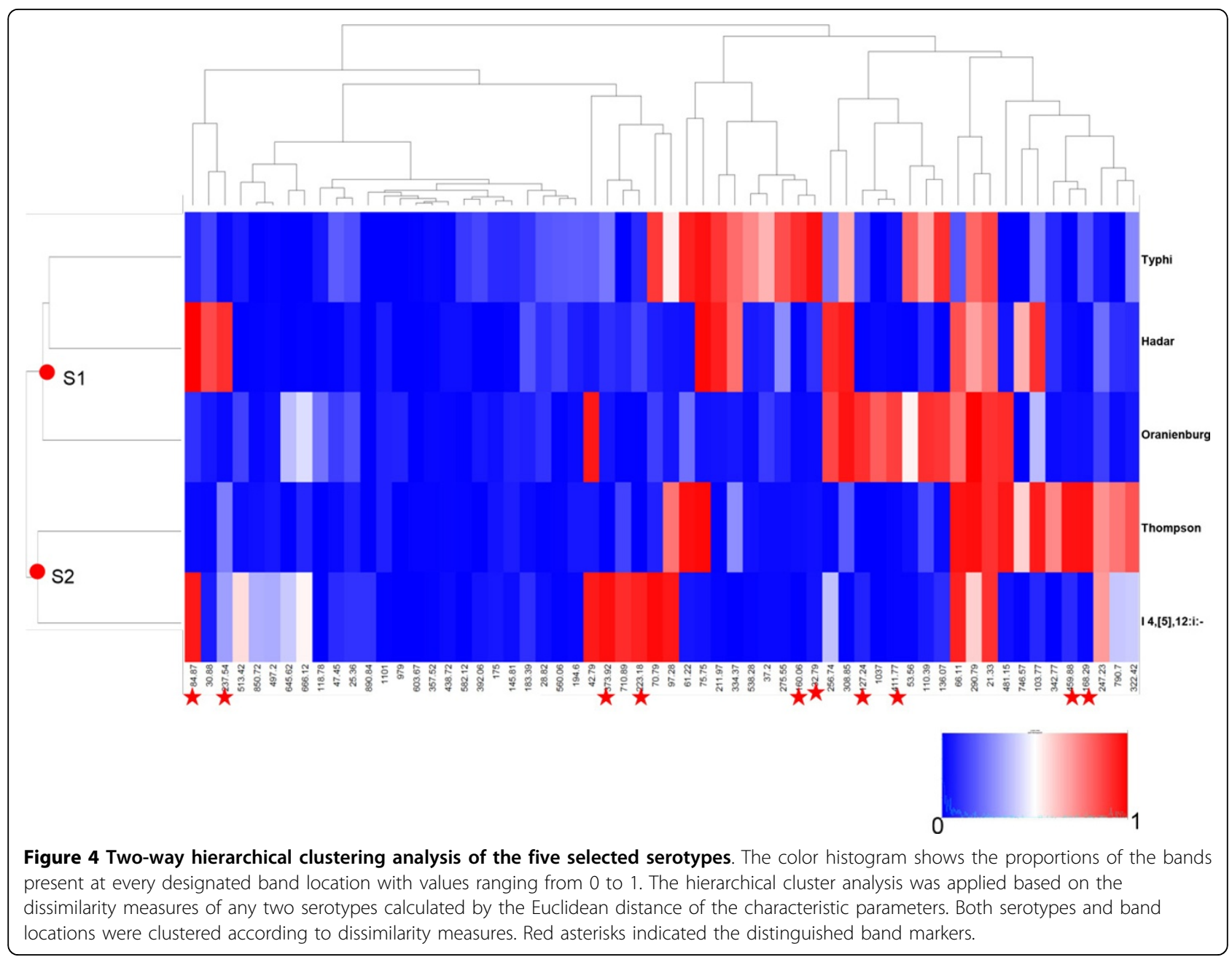

patterns of five serotypes retrieved from the database as a case study. The squares in the matrix showed various colors ranging from blue to red, indicating various degrees of similarity of patterns within every pair of serotypes. Five blue squares in the diagonal, which were distinguishable from the other squares, represent the close distances between the various patterns within the same serotype (Figure 3 ). The rest of the squares were red or $\mathrm{red} /$ white, indicating the distances between the patterns of their corresponding horizontal and vertical serotypes. The bright blue square of serotype Thompson indicated that 2,045 patterns of this serotype were similar to each other; while serotype Hadar showed close relationship with serotype Thompson and Typhi (pale red/white squares). The result was concordant with the prediction accuracies [21] and hierarchical cluster analysis (Figure 2). Users can directly visualize the similarities/dissimilarities of any two individual patterns and the inter- and intra-serotype relationships of two or more serotypes by using this distance matrix analysis tool with the bound PFGE database.
The tool of two-way hierarchical cluster analysis provides the summary of the overall relationships between selected serotypes as well as the distinguishable band markers of these serotypes. Using this tool, the hierarchical cluster analysis is applied to the dissimilarity measures of any pair of the selected serotypes calculated by the Euclidean distance of the characteristic parameters. The color of each of the blocks from blue to red represents the various average proportions (between 0 and 1) of band occurrences for each of the selected serotypes (Figure 4). As a case study, the tool was applied to the dataset of 10,193 PFGE patterns of five serotypes retrieved from the database, and the result was shown in Figure 4, where both serotypes and band locations were grouped to simultaneously identify the associations between serotypes and bands. The five serotypes were divided into two groups (S1 and S2). The cluster image indicated a group of marker bands (highlighted with red asterisks) which distinguish the five serotypes (Table 2). The tool shows more advantages in identification of serotype relationships when more serotypes are selected. Our 
previous research applied this tool to a meta-analysis of 32 serotypes and reported the close relationship of PFGE patterns between serotypes Hadar to Infantis and Muenchen to Newport [20].

The five functional tools were assembled and integrated into a software package to study PFGE profiles for better understanding the genetic diversity of Salmonella and other foodbornbe pathogens. The analysis tools included in the package allow the systematic analysis of PFGE data from various aspects and make it available to meta-analyze PFGE profiles from large data sets. The software package is currently available in the NCTR internal BACPAK knowledgebase. BACPAK, as a general-purposed bioinformatics pipeline for foodborne pathogen analysis, will be a new addition to the FDA bioinformatics tools at http://www.fda.gov/ScienceResearch/BioinformaticsTools/default.htm.

\section{Conclusions}

Although NGS and other sequencing technologies are advancing rapidly in foodborne pathogen subtyping, PFGE is still the most widely used method to characterize Salmonella strains isolated from outbreaks[30]. In the developed software package, PFGE band standardization normalizes the data for cross-group large dataset analysis. The Salmonella serotype prediction tool based on PFGE patterns allows rapid and accurate prediction of Salmonella serotypes from outbreaks before the conventional serological methods are pursued. It also shows advantages in distinguishing an isolate that is serotyped as "unknown" by conventional methods, or for a laboratory where standard serotyping is not available. Hierarchical cluster analysis could be used to clarify the subsets of a group of PFGE patterns for source tracking and identification of outbreak isolates. Since Salmonella serotypes can be closely related in terms of their virulence, and antimicrobial resistance profiles [17,30-33], our distance matrix analysis and twoway hierarchical analysis tools make it possible to study the relationships between phenotypes and genotypes of Salmonella isolates and to distinguish band markers and PFGE pattern diversity for serotype identification, especially for large dataset analysis. Theoretically, these approaches could be applied to other gel-based analysis and other pathogens in the future. Combined with the Salmonella genome sequencing data, the distinct serotype specific patterns and bands may provide useful information to aid in the distribution of serotypes in the population and potentially reduce the need for laborious analyses, such as traditional serotyping. In addition, the PFGE analysis tools in the software package are expected to help the in silico pattern construction to match PFGE data with NGS data in future studies.

\section{Competing interests}

The authors declare that they have no competing interests.

\section{Authors' contributions}

WL, HC, WZ (Zhao) and WZ performed all calculations and data analysis, and WZ wrote the first draft of manuscript. WZ and JC developed the methods and had the original idea and guided the data analysis and presentation of results. WZ, SF and RN collected and generated data, and HT, JM and HF constructed the database and computerized the scripts into practical tools. All authors contributed in data verification, and approach evaluation and assisted with writing the manuscript. All authors read and approved the final manuscript.

\section{Acknowledgements}

This work and the publication were funded by Food Protection Plan of FDA. We are grateful to Ms. Beth Juliar and Dr. Tzu-Pin Lu for critical reading of this manuscript.

This article has been published as part of BMC Bioinformatics Volume 14 Supplement 14, 2013: Proceedings of the Tenth Annual MCBIOS Conference. Discovery in a sea of data. The full contents of the supplement are available online at http://www.biomedcentral.com/ bmcbioinformatics/supplements/14/S14.

\section{Authors' details}

'Division of Bioinformatics and Biostatistics, U.S. Food and Drug Administration, Jefferson, Arkansas, USA. ²Division of Microbiology, U.S. Food and Drug Administration, Jefferson, Arkansas, USA. ${ }^{3}$ Department of Applied Mathematics, Feng Chia University, Taichung, Taiwan. ${ }^{4}$ Graduate Institute of Biostatistics and Biostatistics Center, China Medical University, Taichung, Taiwan. ${ }^{5}$ The Office of Scientific Coordination, National Center for Toxicological Research, U.S. Food and Drug Administration, Jefferson, Arkansas, USA.

\section{Published: 9 October 2013}

\section{References}

1. Scallan E, Hoekstra RM, Angulo FJ, Tauxe RV, Widdowson MA, Roy SL, Jones JL, Griffin PM: Foodborne illness acquired in the United Statesmajor pathogens. Emerg Infect Dis 2011, 17(1):7-15.

2. CDC: Salmonella: annual summary, 2006. CDC, Division of Foodborne, Bacterial and Mycotic Diseases Atlanta, GA.

3. Wattiau P, Boland C, Bertrand S: Methodologies for Salmonella enterica subsp. enterica Subtyping: Gold Standards and Alternatives. Appl Environ Microbiol 2011, 77(22):7877-7885.

4. Grimont PAD, Weill FX: Antigenic Formulae of the Salmonella Serovars. World Health Organization Collaborating Center for Reference and Research on Salmonella. Institut Pasteur, Paris, France; 92007.

5. Kerouanton A, Marault M, Lailler R, Weill FX, Feurer C, Espie E, Brisabois A: Pulsed-field gel electrophoresis subtyping database for foodborne Salmonella enterica serotype discrimination. Foodborne Pathog Dis 2007, 4(3):293-303.

6. Kidgell C, Reichard U, Wain J, Linz B, Torpdahl M, Dougan G, Achtman M: Salmonella typhi, the causative agent of typhoid fever, is approximately 50,000 years old. Infect Genet Evol 2002, 2(1):39-45.

7. Stepan RM, Sherwood JS, Petermann SR, Logue CM: Molecular and comparative analysis of Salmonella enterica Senftenberg from humans and animals using PFGE, MLST and NARMS. BMC Microbio/ 2011, 11:153.

8. Beranek A, Mikula C, Rabold P, Arnhold D, Berghold C, Lederer I, Allerberger F, Kornschober C: Multiple-locus variable-number tandem repeat analysis for subtyping of Salmonella enterica subsp. enterica serovar Enteritidis. Int J Med Microbiol 2009, 299(1):43-51.

9. Chiou CS, Hung CS, Torpdahl M, Watanabe H, Tung SK, Terajima J, Liang SY, Wang YW: Development and evaluation of multilocus variable number tandem repeat analysis for fine typing and phylogenetic analysis of Salmonella enterica serovar Typhimurium. Int J Food Microbiol 2010, 142(1-2):67-73.

10. Allard MW, Luo Y, Strain E, Li C, Keys CE, Son I, Stones R, Musser SM, Brown EW: High resolution clustering of Salmonella enterica serovar Montevideo strains using a next-generation sequencing approach. BMC Genomics 2012, 13:32. 
11. Cao G, Zhao S, Strain E, Luo Y, Timme R, Wang C, Brown E, Meng J, Allard M: Draft genome sequences of eight Salmonella enterica serotype newport strains from diverse hosts and locations. J Bacteriol 2012, 194(18):5146.

12. Lienau EK, Strain E, Wang C, Zheng J, Ottesen AR, Keys CE, Hammack TS, Musser SM, Brown EW, Allard MW, et al: Identification of a salmonellosis outbreak by means of molecular sequencing. N Engl J Med 2011, 364(10):981-982.

13. Hoffmann M, Zhao S, Luo Y, Li C, Folster JP, Whichard J, Allard MW, Brown EW, McDermott PF: Genome sequences of five Salmonella enterica serovar Heidelberg isolates associated with a 2011 multistate outbreak in the United States. J Bacteriol 2012, 194(12):3274-3275.

14. Timme RE, Allard MW, Luo Y, Strain E, Pettengill J, Wang C, Li C, Keys CE, Zheng J, Stones R, et al: Draft genome sequences of 21 Salmonella enterica serovar enteritidis strains. J Bacteriol 2012, 194(21):5994-5995.

15. Elkins CA, Kotewicz ML, Jackson SA, Lacher DW, Abu-Ali GS, Patel IR: Genomic paradigms for food-borne enteric pathogen analysis at the USFDA: case studies highlighting method utility, integration and resolution. Food Addit Contam Part A Chem Anal Control Expo Risk Assess 2012

16. Barrett TJ, Gerner-Smidt P, Swaminathan B: Interpretation of pulsed-field gel electrophoresis patterns in foodborne disease investigations and surveillance. Foodborne Pathog Dis 2006, 3(1):20-31.

17. Gerner-Smidt P, Hise K, Kincaid J, Hunter S, Rolando S, Hyytia-Trees E, Ribot EM, Swaminathan B: PulseNet USA: a five-year update. Foodborne Pathog Dis 2006, 3(1):9-19.

18. Swaminathan B, Barrett TJ, Hunter SB, Tauxe RV: PulseNet: the molecular subtyping network for foodborne bacterial disease surveillance, United States. Emerg Infect Dis 2001, 7(3):382-389.

19. Ribot EM, Fair MA, Gautom R, Cameron DN, Hunter SB, Swaminathan B, Barrett TJ: Standardization of pulsed-field gel electrophoresis protocols for the subtyping of Escherichia coli 0157:H7, Salmonella, and Shigella for PulseNet. Foodborne Pathog Dis 2006, 3(1):59-67.

20. Zou W, Chen HC, Hise KB, Tang H, Foley SL, Meehan J, Lin WJ, Nayak R, $\mathrm{Xu} J$, Fang $\mathrm{H}$, et al: Meta-analysis of pulsed-field gel electrophoresis fingerprints based on a constructed Salmonella database. PLoS One 2013, 8(3):e59224

21. Zou W, Lin WJ, Hise KB, Chen HC, Keys C, Chen JJ: Prediction system for rapid identification of Salmonella serotypes based on pulsed-field gel electrophoresis fingerprints. J Clin Microbiol 2012, 50(5):1524-1532.

22. Breiman L: Random Forests. Machine Learning 2001, 45(1):5-32.

23. Vapnik V: The Nature of Statistical Learning Theory. New York: Springer; 1995.

24. Zou W, Lin WJ, Foley SL, Chen CH, Nayak R, Chen JJ: Evaluation of pulsedfield gel electrophoresis profiles for identification of Salmonella serotypes. J Clin Microbiol 2010, 48(9):3122-3126.

25. Murtagh F: Lectures in Computational Statistics: Multidimensional Clustering Algorithms (Compstat Lectures, No 4). Springer-Verlag; 1985.

26. Jaccard P: Étude comparative de la distribution florale dans une portion des Alpes et des Jura. Bulletin de la Société Vaudoise des Sciences Naturelles 1901, 37:547-579.

27. Deza EDMM: Encyclopedia of Distances. Springer; 2009.

28. CDC: Salmonella Annual Summary Tables 2009. CDC, Division of Foodborne, Bacterial and Mycotic Diseases Atlanta, GA

29. Wonderling L, Pearce R, Wallace FM, Call JE, Feder I, Tamplin M, Luchansky JB: Use of pulsed-field gel electrophoresis to characterize the heterogeneity and clonality of Salmonella isolates obtained from the carcasses and feces of swine at slaughter. Appl Environ Microbiol 2003, 69(7):4177-4182

30. Gerner-Smidt $P$, Whichard JM: Foodborne disease trends and reports. Foodborne Pathog Dis 2010, 7(6):609-611.

31. Zou W, Frye JG, Chang CW, Liu J, Cerniglia CE, Nayak R: Microarray analysis of antimicrobial resistance genes in Salmonella enterica from preharvest poultry environment. J Appl Microbiol 2009, 107(3):906-914.

32. Zou W, Al-Khaldi SF, Branham WS, Han T, Fuscoe JC, Han J, Foley SL, Xu J, Fang $\mathrm{H}$, Cerniglia $\mathrm{CE}$, et al: Microarray analysis of virulence gene profiles in Salmonella serovars from food/food animal environment. J Infect Dev Ctries 2011, 5(2):94-105.
33. Frye JG, Lindsey RL, Meinersmann RJ, Berrang ME, Jackson CR, Englen MD, Turpin JB, Fedorka-Cray PJ: Related antimicrobial resistance genes detected in different bacterial species co-isolated from swine fecal samples. Foodborne Pathog Dis 2011, 8(6):663-679.

doi:10.1186/1471-2105-14-S14-S15

Cite this article as: Zou et al:: Data mining tools for Salmonella characterization: application to gel-based fingerprinting analysis. BMC Bioinformatics 2013 14(Suppl 14):S15.

\section{Submit your next manuscript to BioMed Central and take full advantage of:}

- Convenient online submission

- Thorough peer review

- No space constraints or color figure charges

- Immediate publication on acceptance

- Inclusion in PubMed, CAS, Scopus and Google Scholar

- Research which is freely available for redistribution

Submit your manuscript at www.biomedcentral.com/submit
C) Biomed Central 